\title{
Herausforderungen durch neue Konzepte
}

Jahrzehntelang währt schon der Streit zwischen Datenschutz und praxisgerechter Implementierung von Anwendungen. Begleitet wurde er politisch von Rechtsetzungen zum Datenschutz, die vom ersten deutschen Datenschutzgesetz über die 1995 erlassene EU-Richtlinie 95/46/EG (Datenschutzrichtlinie) zum Schutz natürlicher Personen bei der Verarbeitung personenbezogener Daten und zum freien Datenverkehr bis zur DSGVO reicht. Deren sichtbare Auswirkung für den Nutzer ist die Einverständniserklärung zur Verwendung von Cookies. Im Normalfall ein mechanistisches Ritual ohne dass Transparenz für die Konsequenzen geboten wird. Aber auch die DSGVO hat nicht zu einer europaweit einheitlichen Auslegung von bestimmenden Elementen der IT-Systeme geführt. Während sich in Deutschland die Ablehnung eines einheitlichen Personenidentifikationsmerkmals zu einem Quasiverbot entwickelt hat, haben andere EU-Mitgliedsstaaten die Rationalisierungspotentiale dieses Merkmals für die IT-Anwendungskonzepte in der öffentlichen Verwaltung und bei der Gestaltung von elektronischen Gesundheitssystemen erkannt und vielfältig angewendet.

In letzter Zeit mehren sich die Anzeichen für einen Wandel der Strategie der Anbieter von Digitalisierungskonzepten in Deutschland. Dafür stehen beispielsweise das am 28. Januar 2021 vom Bundestag verabschiedete, Registermodernisierungsgesetz ${ }^{\prime}$ mit einem einheitlichen Personenidentifikationsmerkmal und die seit Januar 2021 nutzbare elektronische Patientenakte im Gesundheitswesen. Nach dem Bundestag hat am 5. März 2021 auch der Bundesrat der Einführung einer individuellen Identifikationsnummer für Bürgerinnen und Bürger im Kontakt mit der öffentlichen Verwaltung zugestimmt. Die Verwendung der bereits an die Bürgerinnen und Bürger ausgegebenen individuellen SteuerIdentifikationsnummer soll den Datenaustausch künftig eindeutiger und anwenderfreundlicher gestalten.

In beiden Fällen werden - nach langen kontroversen Debatten - Lösungen für systemübergreifende interoperable Anwendungen in den bestehenden heterogenen Netzen der öffentlichen Verwaltung und des Gesundheitswesens versprochen. Letztendlich sind sie auch die Kapitulation vor dem erfolglosen Bemühen die Digitalisierung in diesen Bereichen mit datenschutzgerechten und wenig anwenderfreundlichen, sicheren' Konzepten voran zu bringen.

Deutlich aufgewertet werden hierbei vom Gesetzgeber eine angestrebte Datensouveränität der Nutzer und letztendlich die Verwirklichung ihrer informationellen Selbstbestimmung im besten Sinne des Begriffes.

Der Bundesdatenschutzbeauftragte hat gegen diese Paradigmenwechsel am 28. Mai 2020 (Öffentliche Anhörung im Ausschuss für Gesundheit des 19. Deutschen Bundestages am 27. Mai 2020 zum Entwurf eines Gesetzes zum Schutz elektronischer Pa- tientendaten in der Telematikinfrastruktur [Patienten-Datenschutz-Gesetz]) ${ }^{1}$ zu und am 21. Januar 2021 (zum Registermodernisierungsgesetz) ${ }^{2}$ deutlich Stellung bezogen.

Worum geht es in der öffentlichen Verwaltung? Das Onlinezugangsgesetz (OZG) ${ }^{3}$ von 2017 sieht die Digitalisierung von 575 Verwaltungsdienstleistungen von Bund und Ländern bis Ende 2022 vor. Der Weg dahin stellte sich wegen der heterogenen ITSysteme in Bund, Ländern und Kommunen und bisher fehlender Interoperabilität der isoliert voneinander entwickelten Anwendungen als zunehmend schwierig heraus.

Der Nationale Normenkontrollrat (NKR) sieht das Registermodernisierungsgesetz als ,Meilenstein für die Digitalisierung der öffentlichen Verwaltung. Das Registermodernisierungsgesetz kann bei zügiger Umsetzung zu jährlichen Kosteneinsparungen von 3,8 Milliarden Euro in der Verwaltung und bei Bürgern führen, heißt es im Bericht des NKR. Weitere 2,2 Milliarden Euro Entlastungen könnten für die Wirtschaft realisiert werden, wenn ein entsprechendes Gesetz für Wirtschaftsregister auf den Weg gebracht wird.

Um den Digitalisierungsrückstand Deutschlands gegenüber vielen anderen Staaten deutlich zu verringern, sind jedoch andere Überlegungen notwendig. Es geht um Anwendungen, die für viele Nutzer deutlich erlebbare Vorteile bringen und die auch hinsichtlich des Datenschutzniveaus akzeptiert werden. (Vor Jahren wurden sie als, Killer-Applikationen' bezeichnet.) Sowohl in der öffentlichen Verwaltung als auch im Gesundheitswesen kann eine Umsetzung der neuen Konzepte deutliche Fortschritte unterstützen.

Als Beispiel für ein neues konzeptionelles Vorgehen im Gesundheitswesen sei die Weiterentwicklung der bisher noch nicht vollständig ausgerollten Telematik-Infrastruktur zu ,TI 2.0: Deutschlands moderne Plattform für Digitale Medizin' genannt. Die grundsätzlichen Bedingungen wie Nutzerzentriertheit, Interoperabilität und einen stabilen sicheren Betrieb werden einen Technologiesprung erfordern.

Wie dargestellt ist das Spektrum der Herausforderungen an die Beteiligten die Gesetzgeber, die Anwendungsentwickler, die Datenschutzaufsichtsbehörden und die Vermittler von NutzerWissen und -Vertrauen - groß.

Der Aufruf an Alle: schafft gemeinsam gesamtgesellschaftlich akzeptable Lösungen.

1 https://www.bfdi.bund.de/DE/Infothek/Transparenz/Stellungnahmen/2020/ StgN_Patienten-Datenschutz-Gesetz.html?cms_templateQueryString=elektronische+patientenakte\&cms_sortOrder=score+desc

2 https://www.bfdi.bund.de/DE/Infothek/Transparenz/Stellungnahmen/2020/ StgN_InnenA-Registermodernisierungsgesetz.html?cms_submit=Senden\&cms_templateQueryString=registermodernisierungsgesetz

3 http://www.gesetze-im-internet.de/ozg/OZG.pdf 